TITLE:

\title{
Vortex-induced vibration and its effect on torsional flutter instability in the case of $B / D=4$ rectangular cylinder
}

\section{$\operatorname{AUTHOR}(S)$ :}

Matsumoto, Masaru; Yagi, Tomomi; Tamaki, Hitoshi; Tsubota, Tatsuki

\section{CITATION:}

Matsumoto, Masaru ... [et al]. Vortex-induced vibration and its effect on torsional flutter instability in the case of $B / D=4$ rectangular cylinder. Journal of Wind Engineering and Industrial Aerodynamics 2008, 96(6-7): 971-983

\section{ISSUE DATE:}

2008-06

URL:

http://hdl.handle.net/2433/89637

\section{RIGHT:}

Copyright @ 2007 Elsevier; この論文は出版社版でありません。引用の 際には出版社版をご確認ご利用ください。; This is not the published version. Please cite only the published version. 


\section{Title:}

Vortex-induced vibration and its effect on torsional flutter instability in the case of $B / D=4$ rectangular cylinder

\section{Authors:}

Masaru Matsumoto $^{\text {a),b) }}$, Tomomi Yagi ${ }^{\text {a),b) }}$, Hitoshi Tamaki ${ }^{\text {c) }}$, Tatsuki Tsubota ${ }^{\text {d) }}$

\section{Affiliations:}

a) Department of Civil and Earth Resources Engineering, Kyoto University, Kyotodaigaku-Katsura, Nishikyo-ku, Kyoto 615-8540, Japan

b) Advanced Research Institute of Fluids Science and Engineering, Int'tech Center, Kyoto University, Kyotodaigaku-Katsura, Nishikyo-ku, Kyoto 615-8510, Japan

c) Nagoya Research \& Development Center, Mitsubishi Heavy Industries, Ltd, 1, Takamichi, Iwatsuka-cho, Nakamura-ku, Nagoya 453-8515, Japan

d) Urban Transportation Headquarters, Hankyu Corporation, 1-16-1, Shibata, Kita-ku, Osaka 530-8689, Japan

\section{Keywords:}

Vortex-induced vibration, Kármán vortex, Motion-induced vortex, Torsional Flutter

\section{Corresponding Author:}

Masaru Matsumoto

Department of Civil and Earth Resources Engineering

Kyoto University

Kyotodaigaku-Katsura, Nishikyo-ku, Kyoto 615-8540, Japan

Tel: +81-075-383-3165

Fax: +81-075-383-3168

E-mail: matsu@brdgeng.gee.kyoto-u.ac.jp 


\title{
Vortex-induced vibration and its effect on torsional flutter instability in the case of $\mathrm{B} / \mathrm{D}=4$ rectangular cylinder
}

\author{
Masaru Matsumoto ${ }^{\mathrm{a}, \mathrm{b}}$, Tomomi Yagi ${ }^{\mathrm{a}, \mathrm{b}}$, Hitoshi Tamaki ${ }^{\mathrm{c}}$, Tatsuki Tsubota $^{\mathrm{d}}$ \\ a) Department of Civil and Earth Resources Engineering, Kyoto University, Kyotodaigaku-Katsura, \\ Nishikyo-ku, Kyoto 615-8540, Japan \\ b) Advanced Research Institute of Fluids Science and Engineering, Int'tech Center, Kyoto University, \\ Kyotodaigaku-Katsura, Nishikyo-ku, Kyoto 615-8510, Japan \\ ${ }^{c)}$ Nagoya Research \& Development Center, Mitsubishi Heavy Industries, Ltd, 1, Takamichi, Iwatsuka-cho, \\ Nakamura-ku, Nagoya 453-8515, Japan \\ d) Urban Transportation Headquarters, Hankyu Corporation, 1-16-1, Shibata, Kita-ku, Osaka 530-8689, \\ Japan
}

\begin{abstract}
For two-dimensional rectangular cylinder with the side-ratio of $4(B / D=4, B$ : along-wind length, $D$ : cross-wind length), two different types of vortex-induced excitation, which are motion-induced vortex excitation, i.e. shear layer instability related-vortex excitation, and Kármán vortex excitation, were observed in both heaving and torsional motion. Also, the role of vortex-generation on the torsional flutter instability was experimentally investigated by changing the rotation axis in the state without/with a splitter plate in the wake. The two different kinds of vortex-induced excitations was confirmed in the close reduced wind speed region, and the vortex-induced excitations could play an important role as triggering of torsional flutter instability when Scruton number is small enough. Furthermore, the flow pattern around the cylinder during torsional motion drastically changes between the low and high reduced velocity.
\end{abstract}

\section{Introduction}

Vortex-induced vibration of two-dimensional bluff bodies can be roughly divided into the Kármán vortex type and the motion-induced vortex type. The former one is characterized by the Strouhal number St, which is a non-dimensional Kármán vortex shedding frequency determined by the shape of body section. This vibration can be explained as a lock-in phenomenon between the Kármán vortex frequency and the natural frequency of the structure. Therefore, this vortex-induced vibration occurs at the reduced wind velocity around $V_{\text {res }}=1 /$ St. On the other hand, the latter one is generated by motion-induced vortices due to the shear layer instability. The motion-induced vortex generated at the leading edge of the body travels on the body surface towards the trailing edge with the velocity of about $60 \%$ of approaching flow. When it reaches the trailing edge and coalesces with the secondary vortex from the trailing edge, the vibration occurs. Then, the onset reduced wind velocity of the motion-induced vortex excitation can be explained as the following equations (Shiraishi and Matsumoto, 1982; Shiraishi and Matsumoto, 1983).

Heaving motion:

$V_{\text {on }}=\frac{1}{N} \cdot \frac{1}{0.6} \cdot \frac{B}{D}=\frac{1}{N} \cdot 1.67 \cdot \frac{B}{D}$

Torsional motion:

$V_{\text {on }}=\frac{2}{2 N-1} \cdot \frac{1}{0.6} \cdot \frac{B}{D}=\frac{2}{2 N-1} \cdot 1.67 \cdot \frac{B}{D}$

In these equations, $B$ and $D$ denote along-wind length and cross-wind length of the body, and $\mathrm{N}=1$, 2 and so on. The reason of different reduced velocities in between the heaving mode and the torsional mode can be explained by the different formation timing of the motion-induced vortex at the leading and the trailing edge, as illustrated in Fig. 1. In this figure, the characteristic reduced velocity $V_{c h}$ is defined as $1.67 \mathrm{~B} / \mathrm{D}$, which is derived from Eq. (1) with $\mathrm{N}=1$.

Considering the vortex shedding properties of the rectangular cylinders, the Strouhal number $S t$ becomes discontinuous at the side-ratio $B / D=2.8$ and 6 . These are due to the variation of separation 
flow's reattachment on the body surface. The important point is that the reduced wind velocity $V_{\text {res }}=1 / S t$ and $V_{c h}=1.67 \mathrm{~B} / \mathrm{D}$ have rather close values in $B / D=2.8-6$, which means that the reduced wind velocity regions for the Kármán vortex induced vibration and the motion-induced vortex excitation of heaving motion are almost the same in these side-ratios. Generally, for bluff body cylinders with these side-ratios, the heaving motion-induced vortex excitation occurs and the Kármán vortices interfere with it (Matsumoto et al., 1993). Furthermore, these vortices interfere or interact with the divergent vibration, such as galloping, torsional flutter and so on. Therefore, in this study, a series of wind tunnel tests were conducted with $B / D=4$ rectangular cylinder and then the interactions between the Kármán vortex, the motion induced vortex and the torsional flutter are investigated. In the wind tunnel tests, a 1DOF heaving motion and three kinds of 1DOF torsional motion with different rotational axes are considered to realize various combinations of motion phase between the leading edge and the torsional edge. Furthermore, to suppress the Kármán vortex shedding from the body, a splitter plate was installed in the wake. The splitter plate can disturb interferences between two shear layers separated from the body, and it is commonly used to reduce the Kármán vortex (e.g. Nakamura and Nakashima, 1986; Matsumoto et al., 1993).

\section{Wind tunnel tests}

A rectangular cylinder with $B / D=4(B=300 \mathrm{~mm}$, $D=75 \mathrm{~mm}$ ) was used in the wind tunnel tests. As shown in Fig.2, this model was supported by coil springs in the wind tunnel with four different ways, which are a 1DOF heaving motion and three kinds of 1DOF torsional motion with different rotational axes, and then, the vibration amplitudes were measured for each case. To suppress the Kármán vortex sheddings, the splitter plate was installed in the wake as shown in Fig.2. In general, the length of splitter plate should be comparatively long to obtain sufficient effect and also the separation between the body and the plate must be an important parameter. Then, its length was determined as $3 B$ and it was installed in the wake of $0.1 B$ downstream side from the trailing edge of the cylinder. All of the experiments were conducted under smooth flow condition.
Furthermore, unsteady pressures around the body under forced motion with four different ways as mentioned above were measured. The forced vibration amplitudes were $2 \eta=10 \mathrm{~mm}$ in the heaving motion and $2 \phi=2^{\circ}$ in the torsional motion, and the vibration frequency was $2.5 \mathrm{~Hz}$ for both motions. The surface pressures were measured at 20 pressure taps at the center of the cylinder span. Then, the fluctuating pressure coefficient on each point is determined as non-dimensional fluctuating pressure using the dynamic pressure of approaching flow. Simultaneously, displacement of the body motion was also measured, and then the phase is defined as the delay of the maximum negative pressure on the upper surface from the maximum downward heaving velocity or the maximum clockwise torsional displacement.

Using the unsteady pressure results, the aerodynamic derivatives (Scanlan and Tomko, 1971) for the lift and pitching moment are calculated as follows.

$$
\begin{aligned}
& L=\frac{1}{2} \rho(2 b) U^{2}\left\{k H_{1}{ }^{*} \frac{\dot{\eta}}{U}+k H_{2}{ }^{*} \frac{b \dot{\phi}}{U}+k^{2} H_{3}{ }^{*} \phi+k^{2} H_{4}{ }^{*} \frac{\eta}{b}\right\} \\
& M=\frac{1}{2} \rho\left(2 b^{2}\right) U^{2}\left\{k A_{1} \frac{\dot{\eta}}{U}+k A_{2} \frac{b \dot{\phi}}{U}+k^{2} A_{3}^{*} \phi+k^{2} A_{4}{ }^{*} \frac{\eta}{b}\right\}
\end{aligned}
$$

In these equations, $\eta$ : heaving displacement, $\phi$ : torsional displacement, $b$ : half cord length, $k$ : reduced frequency $(=b \omega / U, \omega$ : circular frequency), and $H_{\mathrm{i}}{ }^{*}, A_{\mathrm{i}}{ }^{*}$ are aerodynamic derivatives.

\section{Heaving and Torsional vortex-induced excitation}

The velocity-amplitude diagram of heaving motion is shown in Fig. 3. In the figure, the responses of cases with and without the splitter plate are plotted. The first heaving vortex-induced excitation appears at a half of the characteristic reduced velocity $V_{c h}=1.67(B / D)$, that is 3.3 approximately. This means that the vortex generated near the leading edge reaches the trailing edge during two cycles of heaving motion. The second vortex-induced excitation appears at the characteristic reduced velocity $V_{c h}$, that is 6.7, which is close to the Kármán vortex resonance reduced velocity $V_{\text {res }}=1 / S t$ ( $\left.S t=0.138\right)$, that is 7.2. It is argued that the second vortex-induced excitation is excited not by Kármán vortex but by the motion-induced vortex. This fact can be explained by that there are only minor changes of 
response when the Kármán vortex was suppressed by the splitter plate. The black circle plots in the reduced velocity $10-12$, which are without splitter plate case, are not pure heaving motion but include rolling motion. In Fig. 4, the unsteady pressure characteristics, which are the fluctuating pressure coefficients and the phase, are shown. From these plots, the Kármán vortex reduces the motion induced vortex, but has almost no effect on the vortex traveling on the body surface.

The velocity-amplitude diagram of torsional motion with rotational axis at the mid-chord is shown in Fig. 5 and the unsteady pressure characteristics in Fig. 6. The first torsional response for both with/without splitter plate cases is motion-induced vortex excitation near the two third of $V_{c h}$. This means that the leading edge vortex moves to the trailing edge in one and half cycle torsional motion with $60 \%$ velocity of approaching flow and coalesces with the vortex generated at the trailing edge. Also, the Kármán vortex excitation appears at the resonance reduced velocity $V_{\text {res }}=1 / \mathrm{St}$, that is 7.2 , for the without splitter plate case. This Kármán vortex excitation is eliminated by installation of a splitter plate, and then torsional flutter occurs at lower reduced velocity range. In other words, the Kármán vortices reduce the generation of torsional flutter. The detail of interaction between the torsional flutter and the vortex will be discussed later. Comparing two phase diagrams in Fig.6, the phase delay becomes larger by the installation of the splitter plate for $V / f_{\phi} D=9.33$, but less for $V / f_{\phi} D=13.33$. Without splitter plate, the Kármán vortex excitation occurs at $V / f_{\phi} D=9.33$, which means that the Kármán vortices enhance the traveling of the separation vortices from the leading edge to the downstream.

The velocity-amplitude diagram of torsional motion with rotational axis at the leading edge is shown in Fig. 7 and the unsteady pressure characteristics in Fig. 8. When the rotational axis is located at the leading edge or the wake, the vortex generation timing of two motion-induced vortices at the leading and the trailing edge becomes the same timing as heaving motion. Thus, the onset reduced velocity of the motion-induced torsional vortex excitation appears at $V_{c h}=1.67(B / D),(1 / 2) V_{c h}$ and so on, as shown in Eq. (1) instead of Eq. (2). The first torsional responses from $(1 / 2) V_{c h}$ must be the motion-induced vortex excitation and the second one from $V / f_{\phi} D=7.0$, which is very close to $V_{c h}$ or $V_{\text {res }}=1 / \mathrm{St}$, seems to be a torsional flutter. However, in the large damping case, which means the large Scruton number case, the torsional flutter turns into the velocity restricted response, which must be the motion-induced type. This fact may indicate that the torsional flutter is triggered by the motion-induced vortices and this interaction will also be discussed later. From the fluctuating pressure diagram in Fig. 8, it becomes clear that the Kármán vortices restrain the motion-induced vortices at the downstream side.

The velocity-amplitude diagram of torsional motion with rotational axis at the wake is shown in Fig. 9 and the unsteady pressure characteristics in Fig. 10. The responses and even the pressure characteristics are almost the same between the cases with or without the splitter plate. Therefore, these two torsional responses, which occurs from $(1 / 2) V_{c h}$ and $V_{c h}$ must be motion-induced vortex excitations.

\section{Torsional flutter and its interaction with vortex-induced excitation}

The aerodynamic derivatives derived from the forced heaving motion and the forced torsional motion with the mid-chord rotational axis, are shown in Fig. 11. Comparing the cases with and without the splitter plate, it becomes clear that the Kármán vortex does not significantly affect the aerodynamic derivatives. But for the case with the splitter plate, it should be noted that there is a discrepancy on the onset torsional flutter reduced velocity between directly obtained from the free vibration tests as denoted in Fig. 3 and based upon $A_{2}{ }^{*}$. The aerodynamic derivatives $A_{2}{ }^{*}$ and $A_{3}{ }^{*}$ for the other rotational axes, are shown in Fig. 12. In these plots, "measured" means the values directly measured by the forced vibration method and "calculated" means the superposed values using derivatives of the mid-chord rotational axis shown in Fig. 11. There are some differences between them, but globally they show good agreement, if the difference of the heaving amplitude is taking into account. Furthermore, the result shown in Fig. 9, which the torsional flutter can be suppressed if the rotational axis is in the wake, can be understood by the negative value of $\mathrm{A}_{2}{ }^{*}$ at high reduced velocity. 
As shown in the previous section, if the rotational axis locates at the leading edge, the vortex plays the role triggering the torsional flutter in the case of small Scruton number (Scruton, 1965) as shown in Fig. 7. This particular vortex must not be the Kármán vortex because of no significant difference in the responses between the cases with and without the splitter plate as shown in Fig. 7. This vortex must be the motion-induced vortex related to the shear layer instability, and this kind of vortex can be produced only at a restricted low reduced velocity range.

Also, from the previous section in the case with rotational axis at the mid-chord as shown in Fig. 5, it was observed that the Kármán vortex reduces the generation of the torsional flutter. However, considering the results of aerodynamic derivatives as shown in Fig. 11, this explanation may not be accurate. Fig. 13 shows the velocity - amplitude damping diagram for the torsional motion with the rotational axis at the mid-chord and with the splitter plate. There is a velocity restricted low damping area around $V / f_{\phi} D=11-12$, which is rather close to $2 V_{c h}$, that is $\mathrm{N}=1$ in Eq. (2). Therefore, this is due to the motion-induced vortex and it can be concluded that the motion-induced vortex can be a trigger of the torsional flutter. Furthermore, the Kármán vortex reduces this motion-induced vortex and then the onset velocity of the torsional flutter becomes higher in consequence.

These facts can be clearly understood from the unsteady pressure characteristics measured at the various reduced velocity and the vorticity contours obtained by CFD (Shimada and Ishihara, 2003) at similar reduced velocities, during steady torsional motion as shown in Fig. 14. The first wide peak of unsteady pressure at the low reduced velocity is the local separation bubble and the second wide peak can be characterized by the motion-induced vortex, which is produced inside the local separation bubble. These should correspond to the dynamic stall vortex and the vortex patch, respectively, for the torsional motion-airfoil at the stalling angle, reported by McAlister and Carr (1978). Therefore, it can be understood that the low speed torsional flutter should be excited by the motion-induced vortex, which was pointed out by von Kármán (1952) subject to the research in the Tacoma Narrows Bridge failure mechanism investigation, and the high speed torsional flutter can be caused by the motion-induced unsteady separation bubble. Going back on the discrepancy of the onset reduced velocities between the directly measured one and the one evaluated from the $A_{2}{ }^{*}$, the forced vibration method might not sufficiently produce the motion-induced vortex, because of the predefined amplitude. Therefore, at the lower reduced velocity range, $A_{2}{ }^{*}$ shows the negative value of Fig. 11, while the torsional flutter is observed in the free vibration test with the splitter plate as shown in Fig. 5.

\section{Conclusions}

The results obtained in this study on the vortex-induced excitation and its interference on the torsional flutter of $\mathrm{B} / \mathrm{D}=4$ rectangular cylinder are summarized as follows:

1. Two different vortex excitations of $2 \mathrm{D}$ rectangular cylinder with $\mathrm{B} / \mathrm{D}=4$ were confirmed.

2. The motion-induced vortex excitation is characterized by the relative motions of the leading edge and the trailing edge of body.

3. The torsional flutter instability is triggered by the motion-induced vortex excitation.

4. The motion-induced vortex can be produced only at the low reduced velocity range and the flow pattern, in consequence, drastically changes at low and high reduced velocity around the cylinder during torsional motion.

\section{Acknowledgements}

The authors would like to acknowledge to Mr. K. Okubo (former graduate student), Kajima Technical Research Institute for his contributions in a series of wind tunnel tests.

\section{References}

Kármán, T.V., 1952, Aerodynamic stability of suspension bridges with special reference to the Tacoma Narrows Bridge, Part III The investigation of models of the original Tacoma Narrows Bridge under the action of wind, by F.B. Farquharson.

Matsumoto, M., Shiraishi, N., Shirato, H., Stoyanoff, S., Yagi, T., 1993, Mechanism of, and turbulence effect on vortex-induced oscillations for bridge box girders, Journal of Wind Engineering and Industrial Aerodynamics, 49, pp.467-476.

McAlister, K.W., Carr, L.W., 1978, Water Tunnel 
Visualization of Dynamic Stall, ASME Publication, Symposium on Nonsteady Fluid Dynamics, Winter Meeting, D. E. Crow, J. A. Miller eds., pp.103-111.

Nakamura, Y., Nakashima, M., 1986, Vortex excitation of prisms with elongated rectangular, $\mathrm{H}$ and $\mathrm{T}$ cross-sections, Journal of Fluid Mechanics, 163, pp.149-169.

Scanlan, R.H., Tomko, J.J., 1971, Airfoil and bridge deck flutter derivatives, Journal of the Engineering Mechanics Division, ASCE, 97, EM6, pp.1717-1737.

Scruton, C., 1965, On the wind-excited oscillations of stacks, towers and masts”, Proceedings of First International Conference on Wind Effects on Buildings and Structures, London, pp.798-836.

Shimada, K., Ishihara, T., 2003, Numerical simulation of vortex-induced vibration and torsional flutter of rectangular cross-sections by k- $\varepsilon$ model, 4th ASME JSME Joint Fluid Engineering Conference, Honolulu, CDROM

Shiraishi, N., Matsumoto, M., 1982, On vortex-induced oscillations of bluff cross sections used for bridge structures, Proceedings of the Japan Society of Civil Engineers, 322, pp.37-50, (in Japanese).

Shiraishi, N., Matsumoto, M., 1983, On classification of vortex-induced oscillation and its application for bridge structures, Journal of Wind Engineering and Industrial Aerodynamics, 14, pp.419-430. 


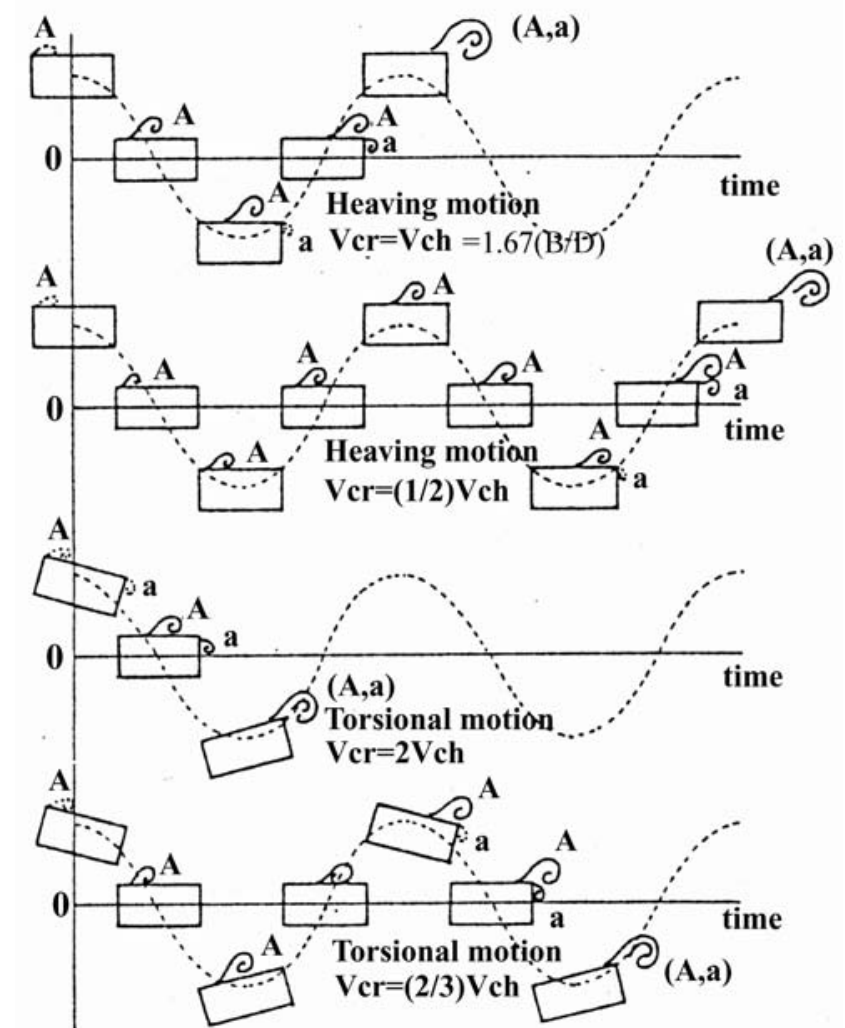

Fig. 1. Motion-induced vortex due to the heaving or the torsional motion.

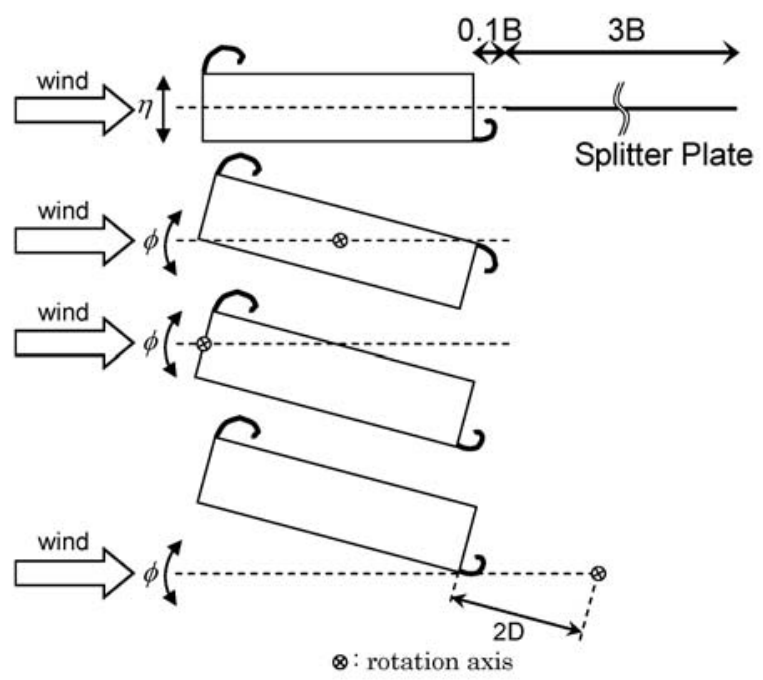

Fig. 2. Heaving motion and torsional motion of $B / D=4$ rectangular cylinder.

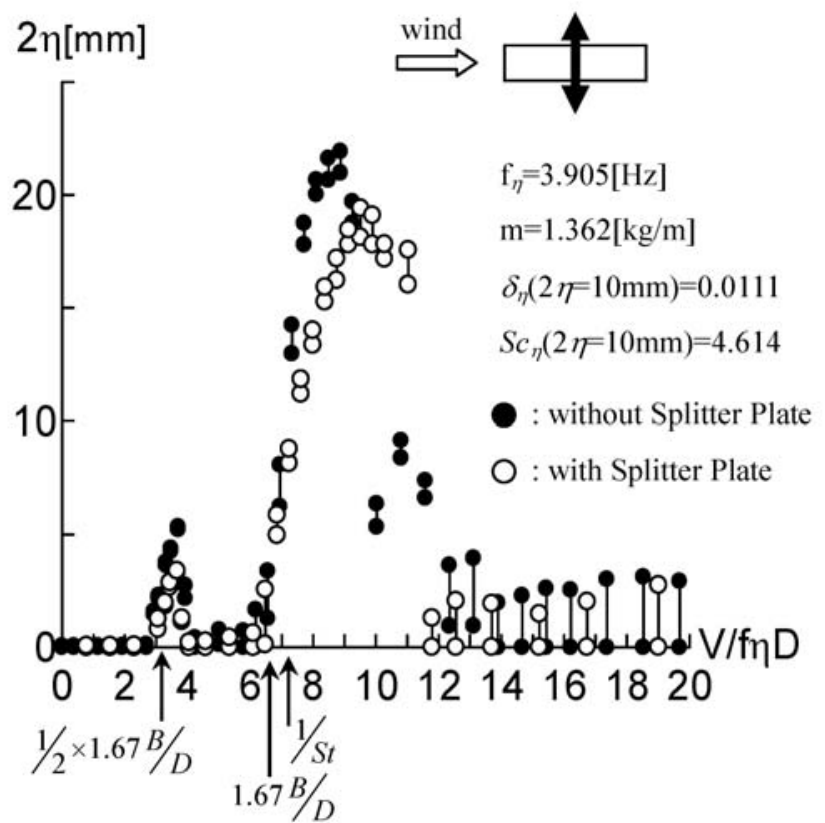

Fig. 3. V-A diagram of heaving motion of $B / D=4$ rectangular cylinder in smooth flow.

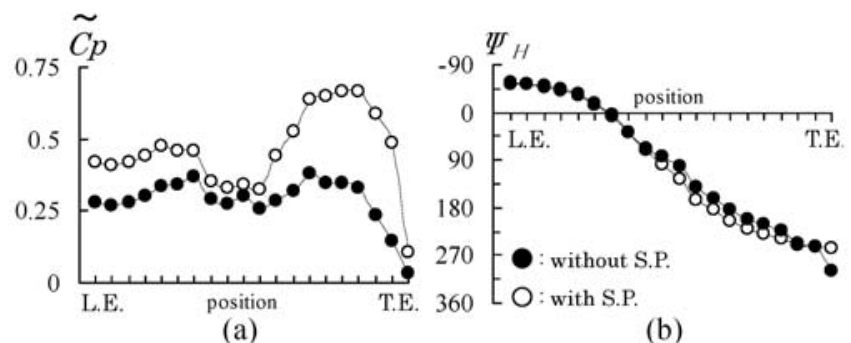

Fig. 4. Fluctuating pressure coefficient diagram (a) and phase diagram (b) of heaving motion of $B / D=4$ rectangular section $\left(V / f_{\eta} D=9.33\right.$, in smooth flow). 


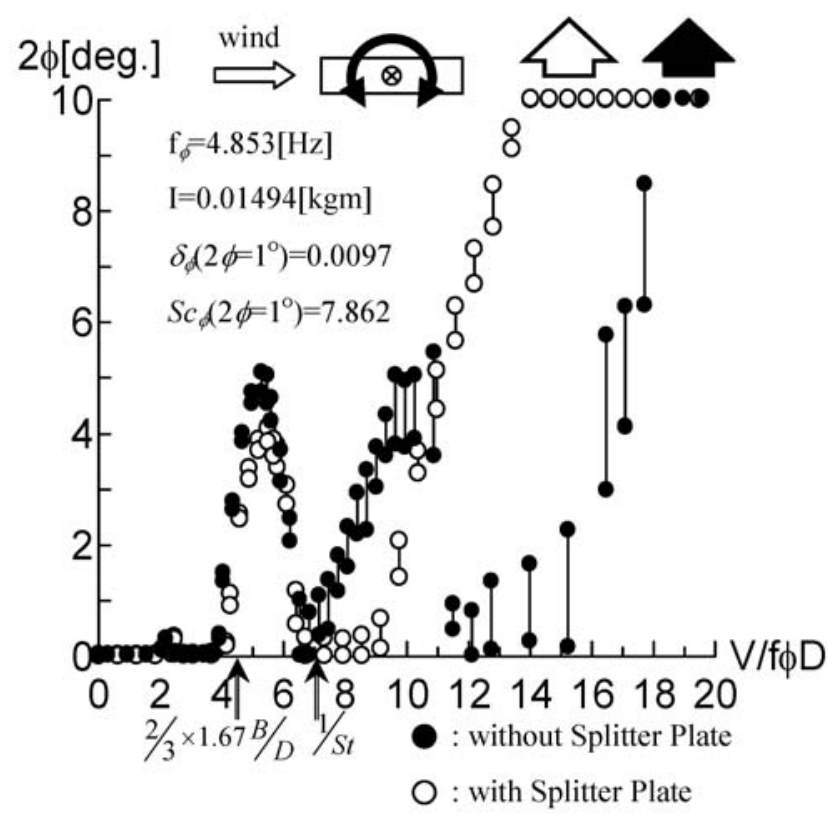

Fig. 5. V-A diagram of torsional motion of $B / D=4$ rectangular cylinder in smooth flow (rotational axis at mid-chord).
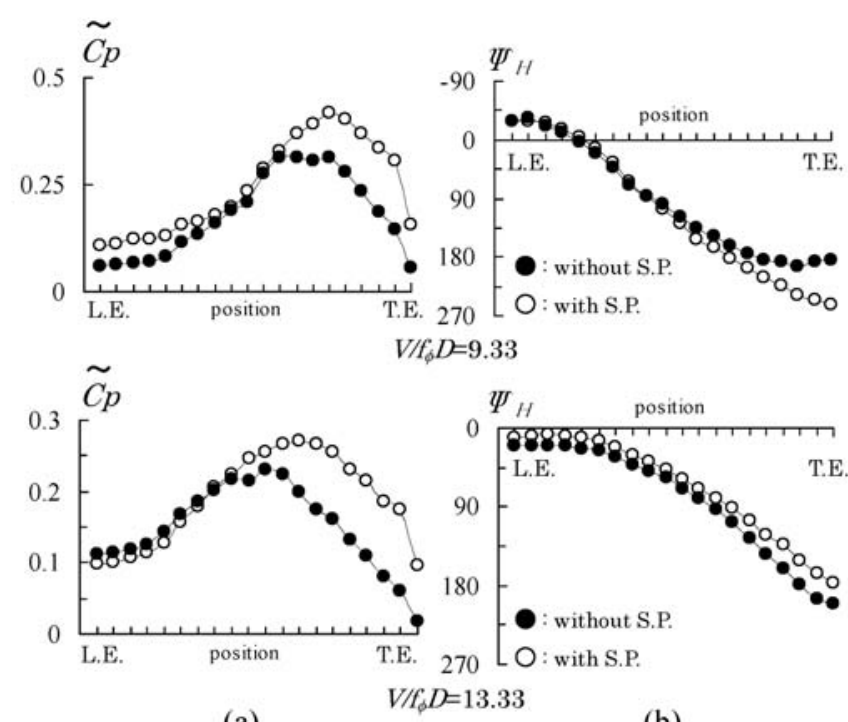

(a)

(b)

Fig. 6. Fluctuating pressure coefficient diagram (a) and phase diagram (b) of torsional motion of $B / D=4$ rectangular section (rotational axis at mid-chord, $V / f_{\phi} D=9.33$ and 13.33, in smooth flow).

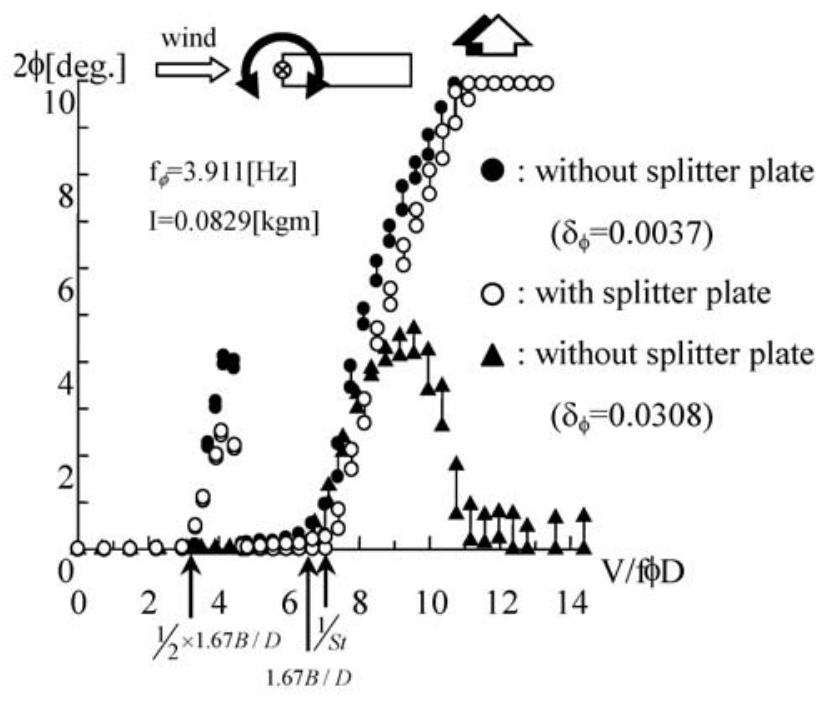

Fig.7. V-A diagram of torsional motion of $B / D=4$ rectangular cylinder in smooth flow (rotational axis at leading edge).
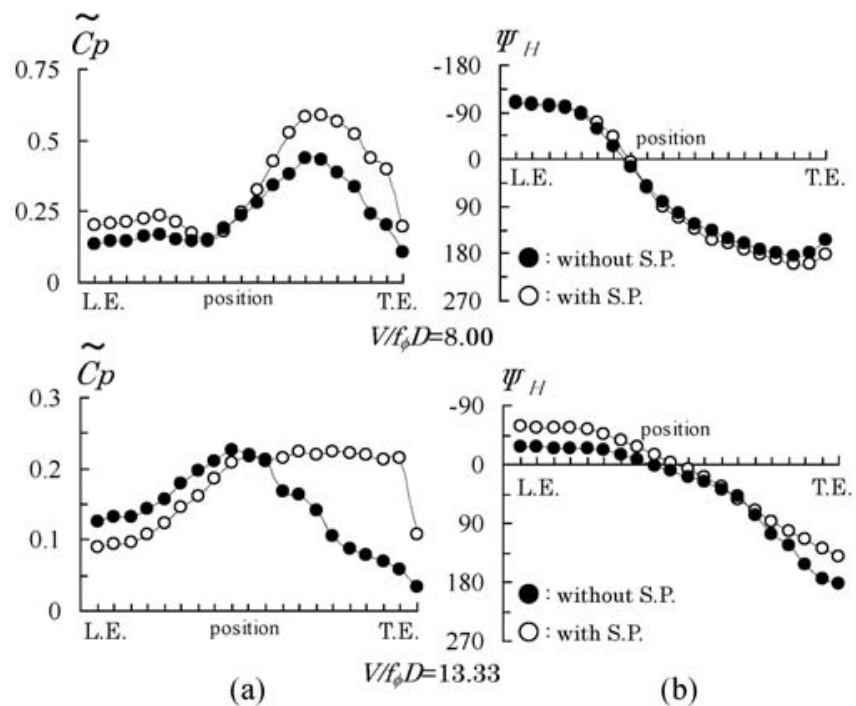

Fig. 8. Fluctuating pressure coefficient diagram (a) and phase diagram (b) of torsional motion of $B / D=4$ rectangular section (rotational axis at leading edge, $V / f_{\phi} D=8.00$ and 13.33, in smooth flow). 


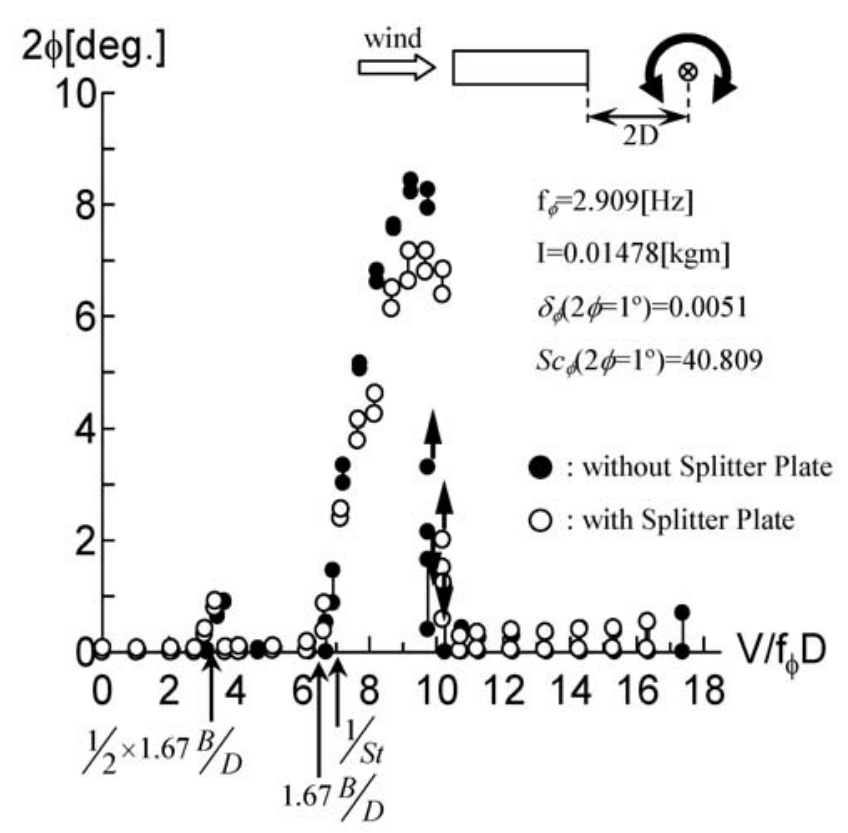

Fig.9. V-A diagram of torsional motion of $B / D=4$ rectangular cylinder in smooth flow (rotational axis at wake).

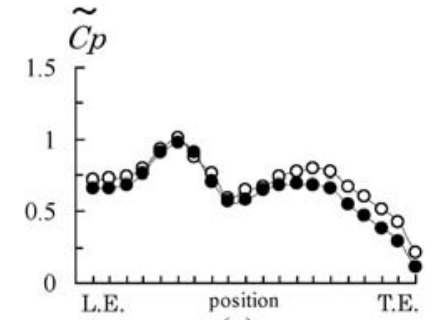

(a)

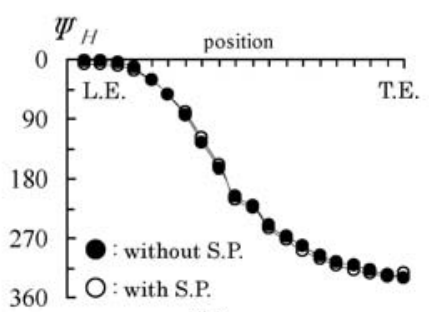

(b)

Fig.10. Fluctuating pressure coefficient diagram (a) and phase diagram (b) of torsional motion of $B / D=4$ rectangular section (rotational axis at wake, $V / f_{\phi} D=9.33$, in smooth flow). 

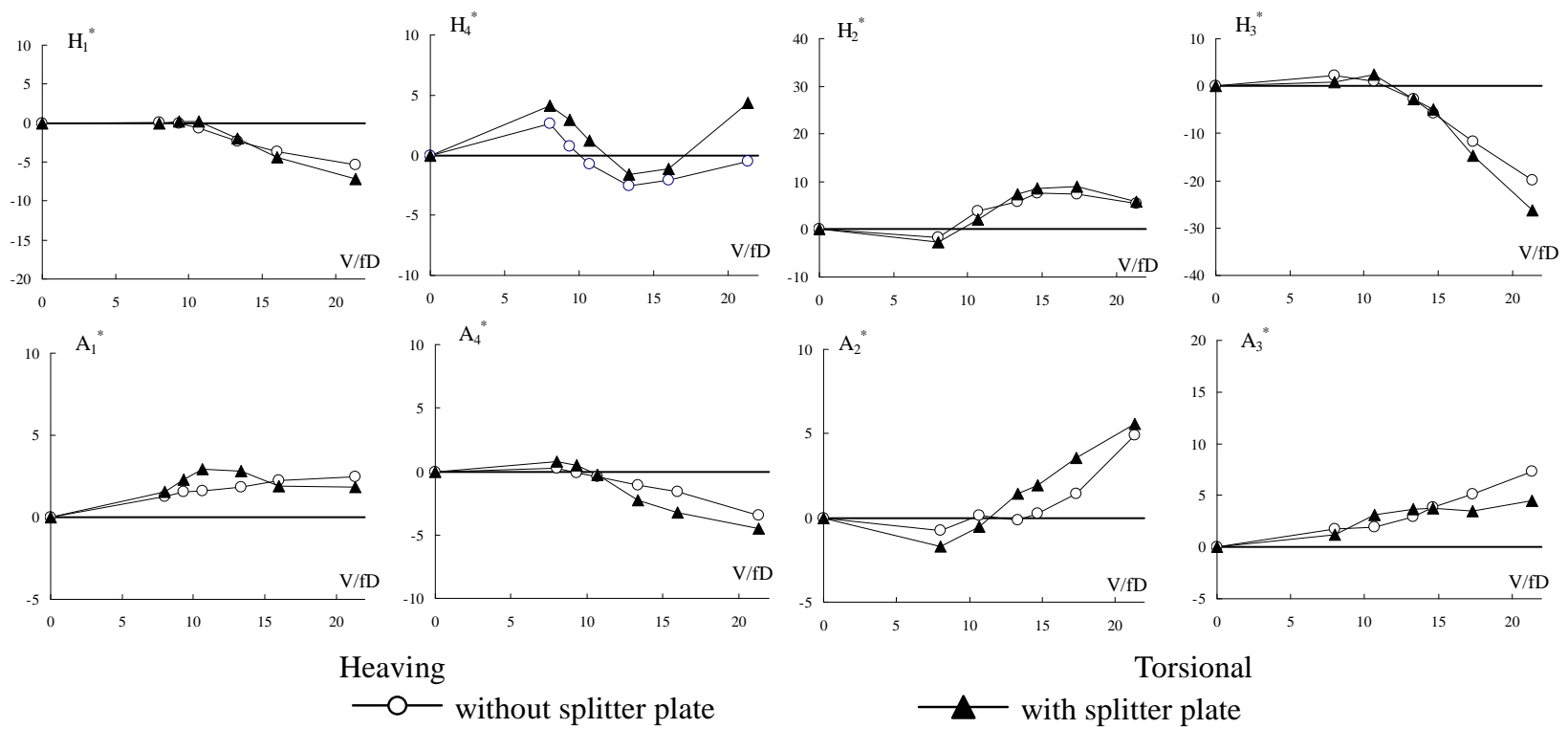

Fig. 11. Aerodynamic derivatives (rotational axis at mid-chord, in smooth flow).
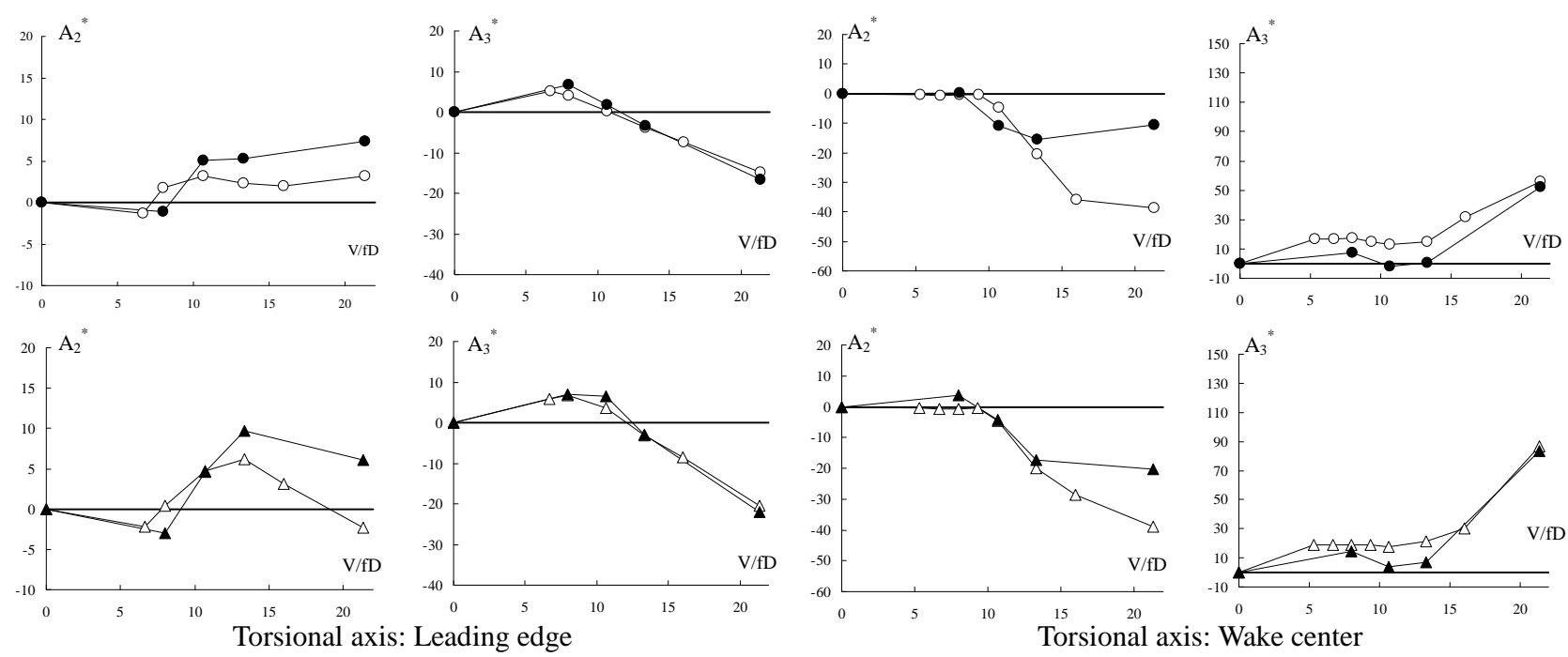

- - without splitter plate(measured)

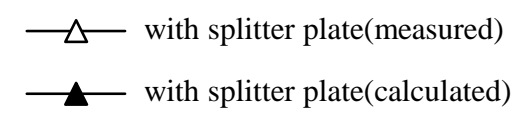

Fig. 12. Aerodynamic derivatives $A_{2}{ }^{*}$ and $A_{3}{ }^{*}$ (rotational axis at leading edge and wake, in smooth flow). 


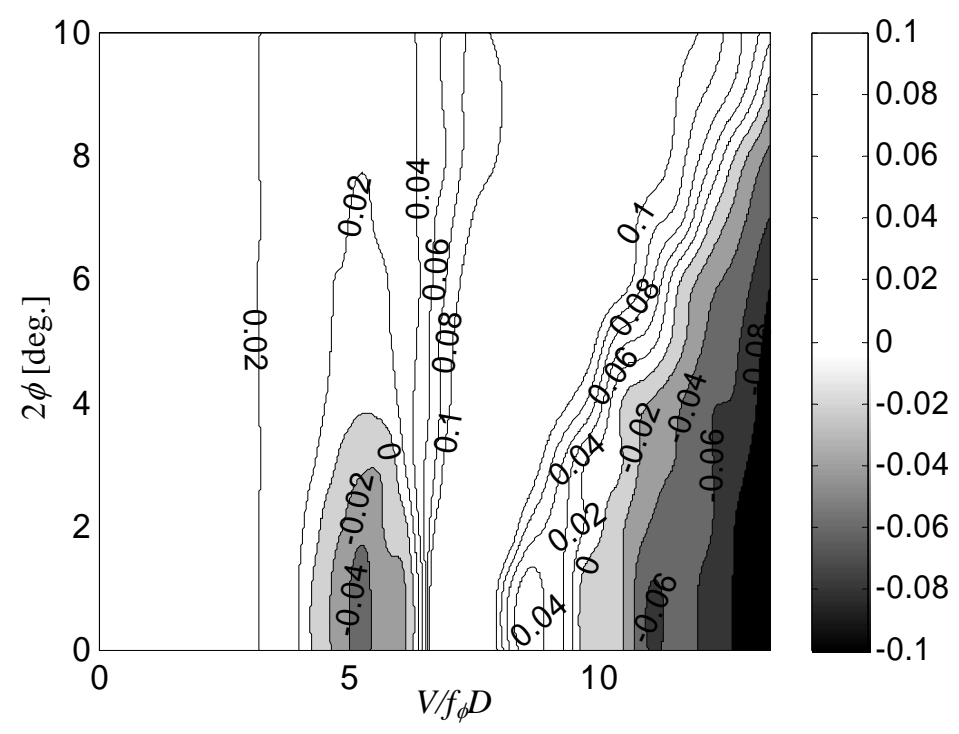

Fig. 13. V-A- $\delta$ diagram of torsional motion of $B / D=4$ rectangular cylinder in smooth flow (rotational axis at mid-chord, with splitter plate). 


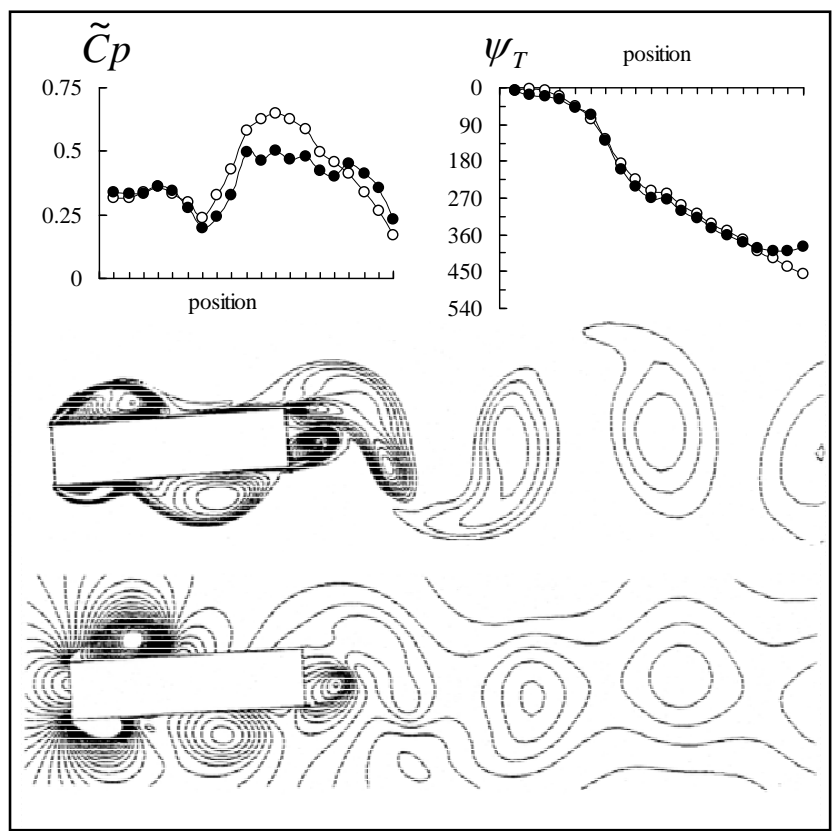

(a) $V / f_{\phi} D=5.33$ (Pressure) \& 5.67(CFD)

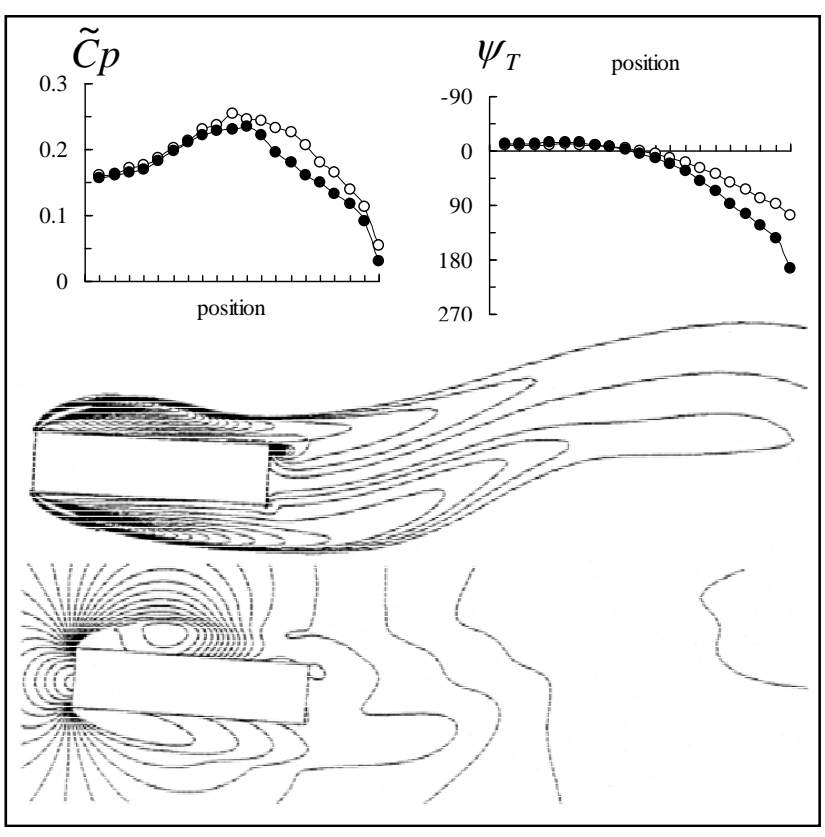

(b) $V / f_{\phi} D=21.33$ (Pressure) $\& 22(\mathrm{CFD}$ )

Fig. 14. Unsteady pressure characteristics and the vorticity contours from CFD (Shimada and Ishihara, 2003) 